\title{
Segmentation of Touching Objects Using Motion Information
}

\author{
Miso $\mathrm{Ju}^{1}$ \\ Computer \& Information Science, Korea University, Sejong, Korea \\ E-mail: misoalth@korea.ac.kr \\ Jaewon Sa ${ }^{a}$, Seoungyup Han $^{\mathbf{b}}$ \\ Computer \& Information Science, Korea University, Sejong, Korea \\ E-mail: ${ }^{a}$ sjwon92@korea.ac.kr; ${ }^{b}$ hansy91@korea.ac.kr

\section{Heegon Kim} \\ Computer \& Information Science, Korea University, Sejong, Korea \\ E-mail: khg860korea.ac.kr
}

\section{Yongwha Chung ${ }^{23}$}

Computer \& Information Science, Korea University, Sejong, Korea

E-mail: ychungyekorea.ac.kr

\section{Daihee Park}

Computer \& Information Science, Korea University, Sejong, Korea

E-mail: dhpark@korea.ac.kr

\begin{abstract}
It is important to segment and track objects automatically in many monitoring applications. When the objects as monitored are close each other, however, it is challenging to segment each object from the touching group. Especially, if the number of touching objects is large, the segmentation accuracy degrades significantly. In this paper, we propose a method of reducing the number of touching objects which should be separated. We first detect objects by using the color information and then extract the motion information of a touching group by using GMM. By excluding the non-moving objects from the touching group, we can reduce the number of touching objects which should be separated. The experimental results show that the proposed method can exclude the non-moving objects from a touching group and thus make the segmentation problem of touching objects liable to be solved.
\end{abstract}

ISCC 2015

18-19, December, 2015

Guangzhou, China

\footnotetext{
${ }^{1}$ Speaker

${ }^{2}$ This research was supported by the Basic Science Research Program through the National Research Foundation of Korea (NRF) funded by the Ministry of Education, Science and Technology (2015R1D1A1A09060594).

${ }^{3}$ Corresponding Author
} 


\section{Introduction}

The object segmentation is very important in many monitoring applications[1,2]., that is, regions of an object segmentation should be uniform and homogeneous with respect to some characteristics (i.e., each region corresponds to each object) so that each object can be identified individually in the monitoring application. Especially, the object tracking in a crowded scene requires accurate segmentation results[3,4]. One of the most widely-used segmentation techniques is a region growing method such as watershed [5-8]; however, it is difficult to separate objects which are close each other with the segmentation methods by using the region growing $[9,10]$. This paper is to develop a new segmentation method to separate the touching objects by using motion information extracted from the input video.

In this study, we assume the livestock monitoring environment with ICT techniques[1115]. In particular, when the number of monitored objects increases, the tracking accuracy in a crowded scene degrades significantly with the increased probability of touching objects[16-18]. In order to solve this problem, we propose a method of reducing the number of touching objects which should be separated. We first detect the objects by using color information and then extract the motion information of a touching group by using Gaussian Mixture Model (GMM) $[19,20]$. By excluding non-moving objects from the touching group, we can reduce the number of touching objects which should be separated. To the best of our knowledge, it is the first report in respect of segmenting the touching objects by using motion information extracted with GMM. Based on the experimental results with OpenCV GMM[21], the proposed method can exclude non-moving objects from a touching group, and thus make the complicated segmentation problem of touching objects liable to be solved (i.e., the number of touching objects to be separated is reduced) with known segmentation solutions such as [22].

The remainder of this paper is organized as follows. Section 2 presents related works with the object segmentation; Section 3 explains the proposed method for simplifying the complicated object segmentation problem by using motion information; Section 4 presents our experimental results; and Section 5 provides a summary of this study.

\section{Related Works}

One of the generally used technique for detecting and tracking individual objects in the stream video data is the method by using frame difference[19,20]. This technique can detect and track objects which are apart from other objects based on the difference of pixels in continuous frames; however, the tracking problem comes up in a way that it mistakes multiple objects for a single object when the images contain two objects or more in close proximity to each other. According to the issue, studies on the automatic separation technique of adjoining objects is on the way briskly.

Various approaches regarding segmentation have been published such as the human cell segmentation algorithm for cell counting and the animal segmentation algorithm for understanding animal's behavior[6.8,17,18,22]. Also, the study of analyzing active mass by tracking athletes at a field house and the research regarding the children's behavior by tracking children's movement at a kindergarten have been conducted [23,24]; however, the segmentation 
problem becomes difficult when the number of touching objects is large. For the sake of accurate segmentation, we need an efficient solution for this complicated segmentation problem.

\section{Segmentation of Touching Objects}

\subsection{Overview of the Proposed Method}

In this study, we propose a method to simplify a complicated problem which is to separate touching objects for precise tracking by using the motion information. We first convert the image in RGB to HSV (Hue Saturation Value) color space, and then binarize the image in order to separate objects from background. We gain motion information from the HSV binary image by applying GMM, and detect moving objects through the information. That is, we exclude nonmoving objects from the motion information. If the detected area of moving objects is larger than single object area, we consider the moving objects as a touching group to be separated.

\subsection{Background Subtraction}

The indoor scenes contain some shadows cast by objects and other noise due to influence of the room lighting. In particular, the size of a shadow is similar to the size of an object, and thus the tracking systems misidentify a single object as two objects due to such noise. In order to solve the misidentification problem, we first transform the image in RGB to HSV color space and adjust the HSV value to eliminate noises such as shadows. Then, we binarize the image to detect objects and subtract background. After performing image binarization, we can obtain the object detection result which is separated from background.

\subsection{Motion Information by Using GMM}

We apply Gaussian Mixture Model (GMM) on the HSV binary image in order to detect the object's behavior and gain the motion information. The OpenCV GMM subtracts background by using background model. To determine the object's motion by using GMM, the learning background and establishing background model is important. The background does not mean the background mentioned in 3.2, but the immovable components for a series of frames. The background model is formed by using each pixel modeled in reference to previous continuous frames.

After the background model is established, we separate the moving objects from the image based on the background model. We gain motion information by the GMM results and detect moving objects. The motion information includes partial-moving objects and full-moving objects. The GMM results eliminate non-moving objects from entire objects, but we identify partial-moving objects with non-moving objects. Accordingly, we count the number of pixels of the detected objects to calculate the size and extract only moving objects based on the size of the object. We set the threshold value based on the size of a single object and eliminate the detected objects when the size of which is smaller than the threshold value. In this way, partialmoving objects are eliminated from the motion information. In addition, if the size of the moving objects detected area is greater than the threshold value, we determine that multiple moving objects are close each other. In other words, the moving objects as detected are in the touching group; however, the GMM results show the potential of misidentifying the entire body 
of corresponding object, and thus we perform the logical AND operation between the background of the touching group and that of the corresponding preprocessed image. After performing the logical AND operation, the foreground touching group which should be separated is extracted.

\section{Experimental Results}

In this paper, we conducted experiments of object segmentation in a pig monitoring application. We set up a camera at $4 \mathrm{~m}$ above on the floor to monitor a pig room and obtained a video of 640480 pixels through the camera. To eliminate some noise, we adjusted the value of HSV color space from image data and generated the HSV binary image as shown in Fig. 1. We can see many touching groups in the HSV binary image. In order to compare segmentation results, we used watershed, one of the generally used segmentation techniques. Fig. 2 shows a segmentation result by applying the watershed to the HSV binary image. On the basis of observation, we can find that some touching groups cannot be segmented correctly.

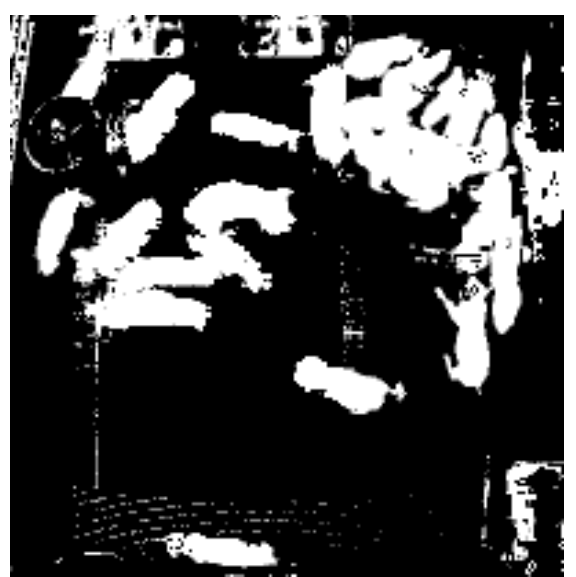

Figure 1: Object Detection Result by Using HSV Color Information.

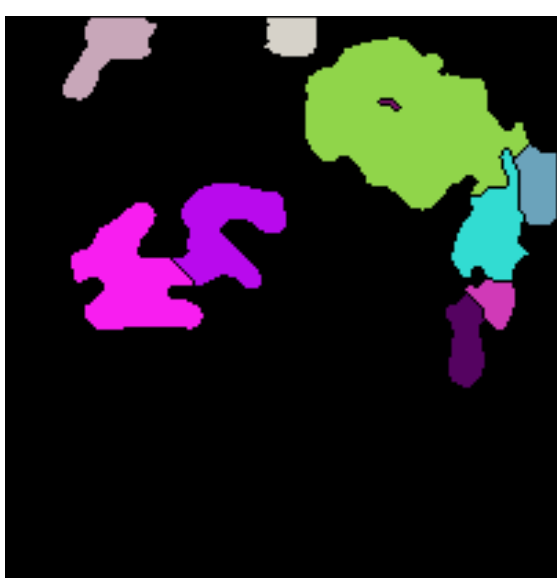

Figure 2: Segmentation Result by Using Watershed on the HSV Binary Image.

After generating the HSV binary image, we extracted the moving pig areas based on motion information which was obtained by applying GMM to the HSV binary image. Fig. 3 shows the detected moving pigs. We could find some small fragments in Fig. 3. These fragments were observed when a pig moved only a part of its body, and thus we regarded them as nonmoving pigs. In the GMM result, the size of a partial body moving was smaller than the threshold value representing the size of a single pig, and thus we obtained the required motion information in separating the moving pigs from the touching group. Finally, we performed the logical AND operation between the background of the minimum rectangle including the moving pigs detected (the red box shown in Fig. 3) and that of the corresponding HSV binary image. After performing the logical AND operation, we could extract the final touching group which should be separated. 


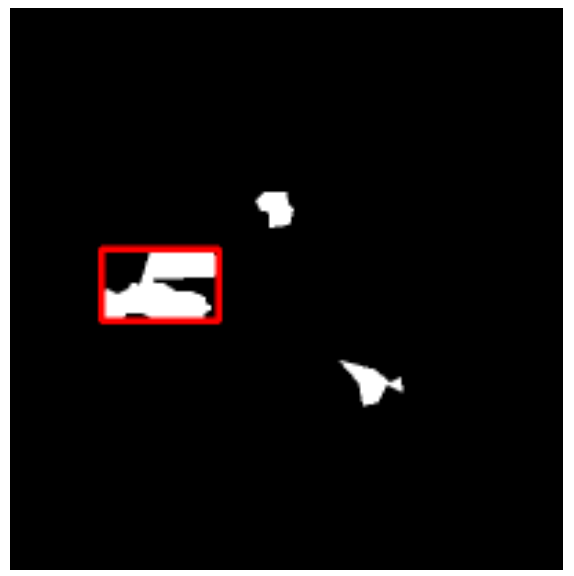

Figure 3: The Motion Detection Result from Using GMM. The red box shows a touching group consisting of moving objects.

The touching pig separation problems become difficult when the number of touching pigs increases; however, if we exploit the motion information, the non-moving pigs shown in the top right corner of Fig. 1 do not need to be separated. In fact, the non-moving pigs were sleeping or taking a rest, and did not need to be separated for tracking. In addition, the separation problem of five touching pigs shown in the middle of Fig. 1 was converted into the separation problem of two touching pigs as shown in Fig. 4. In other words, the proposed method converted a complicated segmentation problem into a manageable problem with a known separation algorithm such as [22] by reducing the number of touching pigs to separate. That is, the proposed method has the advantage of improving the segmentation accuracy significantly by using the motion information. Fig. 5 shows the segmentation result of the final touching objects in Fig. 4 by using the separation algorithm [22]. According to the result image, we confirmed that the segmentation by using the motion information simply separated moving objects in comparison with the existing segmentation techniques (i.e., the segmentation by using the watershed as shown in Fig. 2). Although we assumed a pig monitoring application, we believe the proposed method can be applicable to the general object segmentation applications.

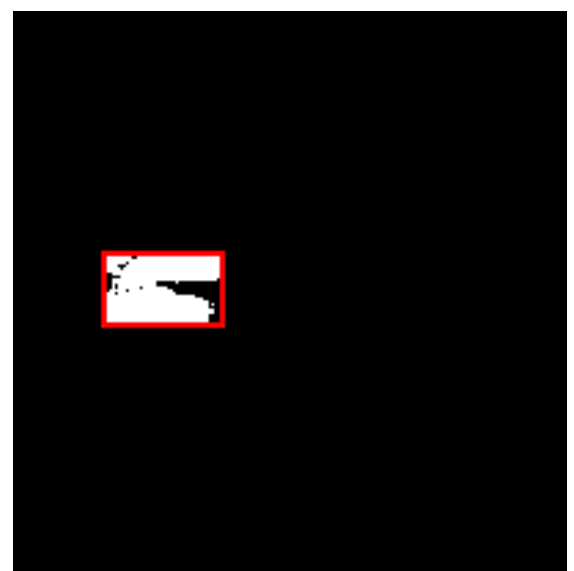

Figure 4: The final touching objects which should be separated.

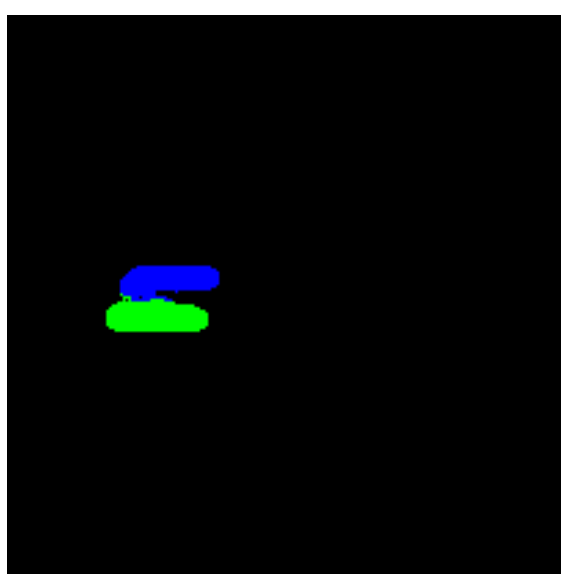

Figure5: Segmentation of the Final Touching Objects. 


\section{Conclusion}

In this study, we proposed a method of reducing the number of touching objects which should be separated from a touching group in a crowded scene. After detecting objects by using the color information, we extracted the motion information of a touching group by using GMM. With this motion information, we could exclude non-moving objects from the touching group. The experimental results showed the proposed method could exclude non-moving objects and thus improve the segmentation accuracy with the known segmentation solutions.

\section{References}

[1] J. Kim and K. Grauman. Shape sharing for object segmentation[M]. Computer Vision-ECCV 2012. $444-458(2012)$

[2] B. Peng, L. Zhang, and D. Zhang. A survey of graph theoretical approaches to image segmentation [J]. Pattern recognition. 46(3): 1020-1038(2013)

[3] S. Dhaliwal and A. Jain. A Survey on Seeded Region Growing based Segmentation Algorithms [J]. International Journal of Computer Science and Management Research. 2(6): 2814-2817(2013)

[4] X. Wang, X. Yang, X. He, Q. Teng, and M. Gao. A high accuracy flow segmentation method in crowded scenes based on streakline[J]. Optik-International Journal for Light and Electron Optics. 125(3): 924-929(2014)

[5] S. T. Rizvi, M. S. Sandhu, and S. E. Fatima. Image Segmentation using Improved Watershed Algorithm[J]. IJCSIT) International Journal of Computer Science and Information Technologies. 5(2): 2543-2545(2014)

[6] H. Tulsani. Segmentation using Morphological Watershed Transformation for Counting Blood Cells[J]. Int. J. Comput. Appl. Inform. Technol. 3(2): 28-36(2013)

[7] V. Grau, A. Mewes, M. Alcaniz, R. Kikinis, and S. Warfield. Improved Watershed Transform for Medical Image Segmentation using Prior Information[J]. IEEE Tr. Med. Imag. 4(23): 447458(2014)

[8] G. de Melo, V. Gomes, C. Baccili, L. de Almeida, and A. Lima. A Robust Segmentation Method for Counting Bovine Milk Somatic Cells in Microscope Slide Images [J]. Computers and Electronics in Agriculture. vol. 115: 142-149(2015)

[9] S. Dalmiya, A. Dasgupta, and S. K. Datta. Application of Wavelet based K-means Algorithm in Mammogram Segmentation[J]. International Journal of Computer Applications. 52(15): 15-19(2012)

[10] W. N. Gonçalves and O. M. Bruno. Automatic System for Counting Cells with Elliptical Shape[J]. Cornell University Library. arXiv preprint arXiv: http://arxiv.org/abs/1201.3109. (2012)

[11] E. S. Nadimi and R. N. Jørgensen. Monitoring and classifying animal behavior using ZigBee-based mobile ad hoc wireless sensor networks and artificial neural networks[J]. Computers and Electronics in Agriculture. vol. 82: 44-54 (2012)

[12] T. Banhazi, H. Lehr, J. Black, H. Crabtree, P. Schofield, M. Tscharke, and D. Berckmans. Precision Livestock Farming: an International Review of Scientific and Commercial Aspects[J]. Int. J. Agric. \& Biol. Eng. 5(3): 1-9(2012)

[13] J. Hwang and H. Yoe. Study on the Smart Application for Wireless Sensor Networks based Ubiquitous Livestock Farm System [J]. Technology. 3(4):

http://weblidi.info.unlp.edu.ar/worldcomp2012-mirror/p2012/ICW2736.pdf (2012) 
[14] A. Nasirahmadi, U. Richter, O. Hensel, S. Edwards, and B. Sturm. Using machine vision for investigation of changes in pig group lying patterns[J]. Computers and Electronics in Agriculture. vol. 119: 184-190(2015)

[15] J. Werner, C. Umstatter, N. Zehner, J. J. Niederhauser, and M. Schick. Validation of a sensor-based automatic measurement system for monitoring chewing activity in horses[J]. Livestock Science, Elsevier. Available online: http://sciencedirect.com. (accessed on 30 July 2015)

[16] Y. Guo, W. Zhu, P. Jiao, C. Ma, and J. Yang. Multi-object extraction from topview group-housed pig images based on adaptive partitioning and multilevel thresholding segmentation[J]. Biosystems Engineering. vol. 135: 54-60(2015)

[17] R. Gronskyte, L. H. Clemmensen, M. S. Hviid, and M. Kulahci. Pig herd monitoring and undesirable tripping and stepping prevention $[\mathrm{J}]$. Computers and Electronics in Agriculture. vol. 119: 51-60(2015)

[18] S. Zuo, L. Jin, Y. Chung, and D. Park. An Index Algorithm for Tracking Pigs in Pigsty[C]. In Proc. of ICITMS, ICIEE 2014 Main Proceedings, Hong Kong. 93: 797-803(2014)

[19] K. Wang, Y. Liang, X. Xing, and R. Zhang. Target Detection Algorithm Based on Gaussian Mixture Background Subtraction Model[C]. In Proc. of the 2015 Chinese Intelligent Automation Conference, Springer, Fuzhou. 336: 439-447(2015)

[20] S. Hatwar, and A. Wanare. GMM based Image Segmentation and Analysis of Image Restoration Techniques[J]. International J. of Computer Applications. 109(16): 45-50(2015)

[21] Open Source Computer Vision. OpenCV. Available online: http://opencv.org. (accessed on 2 May 2015).

[22] Y. Chung, J. Sa, M. Ju, H. Kim, D. Park, and B. Park. Automatic Object Segmentation for Understanding Animal Behavior[C]. Submitted to SMA2016,1-2.

[23] C. B. Santiago, A. Sousa, L. Gomes, L. P. Reis, and L. M. Estriga. Tracking Players in Indoor Sports Using a Vision System Inspired in Fuzzy and Parallel Processing[J]. INTECH Open Access Publisher: 117-140(2012)

[24] R. Sivalingam, A. Cherian, J. Fasching, N. Walczak, N. Bird, V. Morellas, B. Murphy, K. Cullen, K. Lim, G. Sapiro, and N. Papanikolopoulos. A Multi-Sensor Visual Tracking System for Behavior Monitoring of At-Risk Children[C]. IEEE International Conference on Robotics and Automation, USA: 1345-1350(2012). 